\title{
Current status and proposed roles for nitric oxide as a key mediator of the effects of extracellular nucleotides on plant growth
}

\author{
Mari L. Salmi, Greg Clark and Stanley J. Roux* \\ Department of Molecular Biosciences, The University of Texas at Austin, Austin, TX, USA
}

\author{
Edited by: \\ John Hancock, University of the West \\ of England at Bristol, UK \\ Reviewed by: \\ Gary Stacey, University of Missouri, \\ USA \\ Lorenzo Lamattina, Universidad \\ Nacional de Mar del Plata-Consejo \\ Nacional de Investigaciones \\ Científicas y Técnica, Argentina \\ *Correspondence: \\ Stanley J. Roux, Department of \\ Molecular Biosciences, The University \\ of Texas at Austin, 1 University Station \\ A6700, 205 West 24th Street, BIO 16, \\ Austin, TX 78712-0183, USA \\ e-mail: sroux@austin.utexas.edu
}

\begin{abstract}
Recent data indicate that nucleotides are released into the extracellular matrix during plant cell growth, and that these extracellular nucleotides induce signaling changes that can, in a dose-dependent manner, increase or decrease the cell growth. After activation of a presumed receptor, the earliest signaling change induced by extracellular nucleotides is an increase in the concentration of cytosolic $\mathrm{Ca}^{2+}$, but rapidly following this change is an increase in the cellular level of nitric oxide (NO). In Arabidopsis, mutants deficient in nitrate reductase activity (nia1nia2) have drastically reduced nitric oxide production and cannot transduce the effects of applied nucleotides into growth changes. Both increased levels of extracellular nucleotides and increased NO production inhibit auxin transport and inhibit growth, and these effects are potentially due to disruption of the localization and/or function of auxin transport facilitators. However, because NO- and auxin-induced signaling pathways can intersect at multiple points, there may be diverse ways by which the induction of NO by extracellular ATP could modulate auxin signaling and thus influence growth. This review will discuss these optional mechanisms and suggest possible regulatory routes based on current experimental data and predictive computational analyses.
\end{abstract}

Keywords: nitric oxide, extracellular nucleotides, apyrase, auxin transport, post-translational modifications, $S$-nitrosylation, Tyr-nitration, peroxynitrite

\section{INTRODUCTION}

For over 40 years scientists have known that the main energy currency of the cell, ATP, is sometimes released by cells into their extracellular matrix (ECM). In the ECM, ATP functions not primarily to drive energy-dependent reactions, but primarily to bind to receptors and activate signaling changes (Khakh and Burnstock, 2009). The data on this topic have come almost exclusively from studies on animals. However, during the last 10 years an increasing number of reports have demonstrated that signaling changes induced by extracellular ATP (eATP) and other nucleoside triphosphates and diphosphates are a common phenomenon also in plants (Tanaka et al., 2010; Clark and Roux, 2011). In animals, these signaling changes are known to begin with the activation of well-characterized receptors, termed purinoceptors, which fall into two main categories: P2X, which are ion-channel linked, and P2Y, which are G-protein linked. In plants, too, there is strong indirect evidence that there may be at least two kinds of plasma-membrane-localized receptors for extracellular nucleotides (Demidchik et al., 2009, 2011), but their primary structures are clearly different from the animal purinoceptors, and as yet their identity is unknown (Clark and Roux, 2011).

The processes that release ATP and other nucleotides into the ECM are similar in plants and animals and include secretion, active transport, and wound or pathogen events that break the plasma membrane or make it leaky (Roux and Steinebrunner, 2007). Animals and plants also use similar enzymes to limit the build-up of eATP, primarily ecto-nucleoside triphosphate diphosphohydrolases (ecto-NTPDases) or ectoapyrases, which remove the terminal phosphate from nucleoside triphosphate diphosphohydrolases (NTPs) and nucleoside diphosphohydrolases (NDPs; Knowles, 2011).

Although the search to identify plant purinoceptors has not yet yielded definitive results, the signaling changes induced by extracellular nucleotides in animals and plants are already known to be remarkably similar. They begin with a rapid increase in the concentration of free cytosolic calcium $\left(\left[\mathrm{Ca}^{2+}\right]_{\mathrm{cyt}}\right.$; Demidchik etal., 2003, 2009, 2011; Jeter etal., 2004; Burnstock et al., 2010). Afterward, early downstream changes include increased production of superoxide and NO (D'Andrea et al., 2008; Clark and Roux, 2009; Harada, 2010; Tanaka et al., 2010). Sueldo et al. (2010) reported that eATP-induced NO production is downstream of phosphatidic acid production in suspension cultured tomato cells. In plants, mutants that are suppressed in their ability to make either superoxide or nitric oxide are insensitive to the effects of applied nucleotides on cell growth (Clark et al., 2010) and stomatal aperture (Clark et al., 2011; Hao et al., 2012), which suggest these signaling intermediates are needed to convert eATP receptor activation to physiological changes in cells.

This review focuses on the requirement for NO production to transduce extracellular nucleotide signals into growth and other physiological changes in plants. Because there is strong evidence linking extracellular nucleotide effects to changes in auxin transport (Tang etal., 2003; Liu etal., 2012), the question of how 
eATP-induced changes in NO production could alter auxin transport becomes especially relevant. As noted by Liu et al. (2012), current evidence favors post-transcriptional events as being key to changing the molecular activities that drive auxin transport, so this review will especially highlight the role of NO in protein modifications that could rapidly alter either the transport or concentration of auxin in cells.

\section{KINETICS OF SIGNALING RESPONSES INDUCED BY EXTRACELLULAR NUCLEOTIDES}

Two of the better-documented occasions of ATP release by plant cells are wounding (Song et al., 2006) and cell expansion (Kim et al., 2006; Wu etal., 2007; Clark et al., 2011). Wounding, of course, breaks the plasma membrane and allows the leakage of cytoplasmic ATP (concentration $\sim \mathrm{mM}$; Gout et al., 1992) into the ECM. Cell expansion is thought to require the delivery of secretory vesicles to the plasma membrane, and, based on the animal literature, these vesicles can carry up to mM ATP and release it into the ECM upon their fusion with the plasma membrane (Lazarowski et al., 2003). Alternatively, plant cells also release ATP when their membranes are stretched or mechanically stimulated (Jeter et al., 2004; Weerasinghe et al., 2009), and membranes are certainly stretched when plant cells expand. Both wounding and cell expansion would release ATP quickly, and once in the ECM, ATP, and ADP can induce calcium transport changes and thus initiate signal transduction in less than 30 s (Demidchik et al., 2009, 2011).

Downstream of the increase in $\left[\mathrm{Ca}^{2+}\right]_{\text {cyt }}$ induced by extracellular nucleotides are increases in the production of both reactive oxygen species (ROS; e.g., superoxide and $\mathrm{H}_{2} \mathrm{O}_{2}$; Kim et al., 2006; Song et al., 2006; Tonon et al., 2010; Sun et al., 2012) and nitric oxide (Foresi et al., 2007; Wu and Wu, 2008; Reichler et al., 2009; Clark etal., 2010, 2011). Mutant analyses indicate that both of these changes are needed for the growth and other cellular changes induced by extracellular nucleotides (Clark et al., 2010, 2011). Mutants null for the D/F subunit of NADPH oxidase, which catalyzes superoxide production, or nia1nia2 double knockouts, which are null for two genes encoding the nitrate reductase enzyme that accounts for significant fraction of the NO production in root hairs (Clark et al., 2010), do not show growth or stomatal aperture responses to applied nucleotides (Reichler etal., 2009; Clark etal., 2010, 2011). Two recently described signaling mechanisms that might link the eATP-induced increase in $\left[\mathrm{Ca}^{2+}\right]_{\text {cyt }}$ and the activation of NADPH oxidase activity should be evaluated; a calmodulin-domain protein kinase (CDPK), which can activate NADPH oxidase by phosphorylation (Yoshioka et al., 2011), and the NO-mediated regulation of NADOH oxidase by S-nitrosylation (Yoshioka et al., 2011; Yun et al., 2011). The link between increased $\left[\mathrm{Ca}^{2+}\right]_{\text {cyt }}$ and activation of nitrate reductase is not as well documented, although this enzyme activity may also be regulated by phosphorylation (Lea et al., 2004). Wu and Wu (2008) found that eATP $\gamma$ S must induce an increase $\left[\mathrm{Ca}^{2+}\right]_{c y t}$ in hairy roots in order to stimulate NO production. Of course, there are other sources of NO production in plants in addition to nitrate reductase, and there is evidence for cross-talk between these enzymes and $\mathrm{Ca}^{2+}$ signaling (Besson-Bard et al., 2008).
Given the rapidity of eATP release by cells and the need for NO production to transduce the eATP signal into cellular changes, it is surprising that until now the earliest detection of NO production is $10 \mathrm{~min}$ or more after applied nucleotide treatment (Reichler et al., 2009; Clark et al., 2010). This delayed detection may reflect limitations of the assay methods more than actual delay in NO production. Nonetheless, knowing more precisely the kinetics of nucleotide-induced NO production will be important for determining whether NO plays a primary or secondary role in mediating the broad effects of eATP on plant cell growth and physiology, and specifically on auxin transport.

\section{NITRIC OXIDE-DEPENDENT PROTEIN MODIFICATIONS}

Two widely studied post-translational modifications (PTMs) that result from an increase in $\mathrm{NO}$ are nitration of tyrosine residues generating modified 3-nitrotyrosines, and nitrosylation of cysteine residues ( $S$-nitrosylation). Thorough reviews of the proteomic approaches used in plants to identify NO signaling factors (Bykova and Rampitsch, 2013; Jacques et al., 2013; Kovacs and Lindermayr, 2013) and reviews of the various signaling pathways in plants that employ NO-dependent PTM (Astier and Lindermayr, 2012; Corpas et al., 2013) were recently published, so we will not attempt to replicate these. We will focus on what mechanisms link NOmediated modifications to eATP effects on plant growth generally and auxin transport specifically.

To understand this regulation it will be important to confidently identify which proteins are $S$-nitrosylated or Tyr-nitrated, and then evaluate if these modifications play a central role in growth control. A standard method for detecting $S$-nitrosylation of proteins, called the biotin-switch method, relies on NO donor treatment of samples prior to identification of modified proteins (Jaffrey et al., 2001). S-nitrosylation of Arabidopsis proteins was detected by this method after NO-donor treatment in cell suspension culture extracts and leaf tissue (Lindermayr et al., 2005). More recently, a modification of this method that does not rely on application of an NO donor was used to identify endogenously $S$-nitrosylated Arabidopsis proteins, again from cell culture (Fares et al., 2011). Detailed analysis of specific plant proteins modified by $S$-nitrosylation demonstrates that this modification can regulate protein activity (Astier and Lindermayr, 2012; Feng et al., 2013).

The nitrotyrosine PTM has been experimentally detected in proteins of only a few plant systems to date, including 2-week-old Arabidopsis whole seedlings (Lozano-Juste et al., 2011), hypocotyls of 9-day-old sunflowers (Chaki et al., 2009), and pea plants at several different stages of development (Begara-Morales et al., 2013). Each of these studies has demonstrated a regulatory role for the Tyr-nitration observed in at least one protein, as well as identified numerous other targets for this PTM.

Several Arabidopsis proteins that have been experimentally shown to be $S$-nitrosylated or Tyr-nitrated (Lindermayr et al., 2005; Fares et al., 2011; Lozano-Juste et al., 2011) may play a role in eATP signaling pathways because they function in auxin transport or signaling, in ROS signaling, or in wall extensibility. Included in the small number of plant proteins whose regulation by NO has been experimentally validated is the auxin receptor TIR1 (Terrile et al., 2012). This finding indicates that the regulation of growth 
and development by auxin includes, in at least one case, NOdependent PTM of a key protein in auxin signaling, and supports the need to evaluate additional players in this signaling pathway as targets for these regulatory modifications.

There is growing evidence for cross-talk between NO and auxin signaling pathways in root growth and morphology and in responses to iron deficiency (Simontacchi et al., 2013). For example, auxin and $\mathrm{NO}$ are both implicated in heavy metal stress responses (Peto et al., 2011; Xu et al., 2011; Kolbert et al., 2012) and in the formation of both adventitious and lateral roots (Pagnussat et al., 2003, 2004; Correa-Aragunde et al., 2004; Lanteri et al., 2006, 2008; Guo et al., 2008; Liao et al., 2011; Yadav et al., 2011; Li and Jia, 2013). Increasing NO levels in Arabidopsis primary roots results in a decrease of the polar auxin transport mediated by PIN-FORMED 1 (PIN1), and consequent growth inhibition (Fernandez-Marcos et al., 2011). More recently, auxin was suggested to control root morphology by inducing $S$-denitrosylation of an ascorbate peroxidase enzyme involved in redox regulation (Correa-Aragunde et al., 2013). Nitric oxide also plays a role in auxin-induced stomatal opening (She and Song, 2006). Speculatively, this auxin-NO connection could apply also to effects mediated by extracellular nucleotides, as both eATP and apyrase expression influence auxin transport (Tang et al., 2003; Liu et al., 2012), and both regulate stomatal aperture (Clark et al., 2011) and cell growth (Clark et al., 2010) in an NO-dependent manner.

\section{SPECULATION ON POSSIBLE ROLES FOR NITRIC-OXIDE BASED POST-TRANSLATIONAL REGULATION IN EATP RESPONSES}

Unraveling the specific steps of any signaling pathway is a complicated task, because signaling typically occurs in a feedback process wherein well-characterized steps such as increased $\left[\mathrm{Ca}^{2+}\right]_{c y t}$ and protein phosphorylation are the most commonly observed. While studies have shown that both NO-mediated PTMs mentioned above are reversible and can induce physical changes to proteins that regulate their activity (Lindermayr et al., 2005; Lozano-Juste et al., 2011), another interesting possibility that remains to be studied is an indirect regulatory role for these modifications. Bykova

Table 1 | Computationally predicted NO mediated modifications of proteins implicated in eATP signaling (Xue et al., 2010; Liu et al., 2011). Only predictions included in the "high" threshold category are included here (10\% FDR).

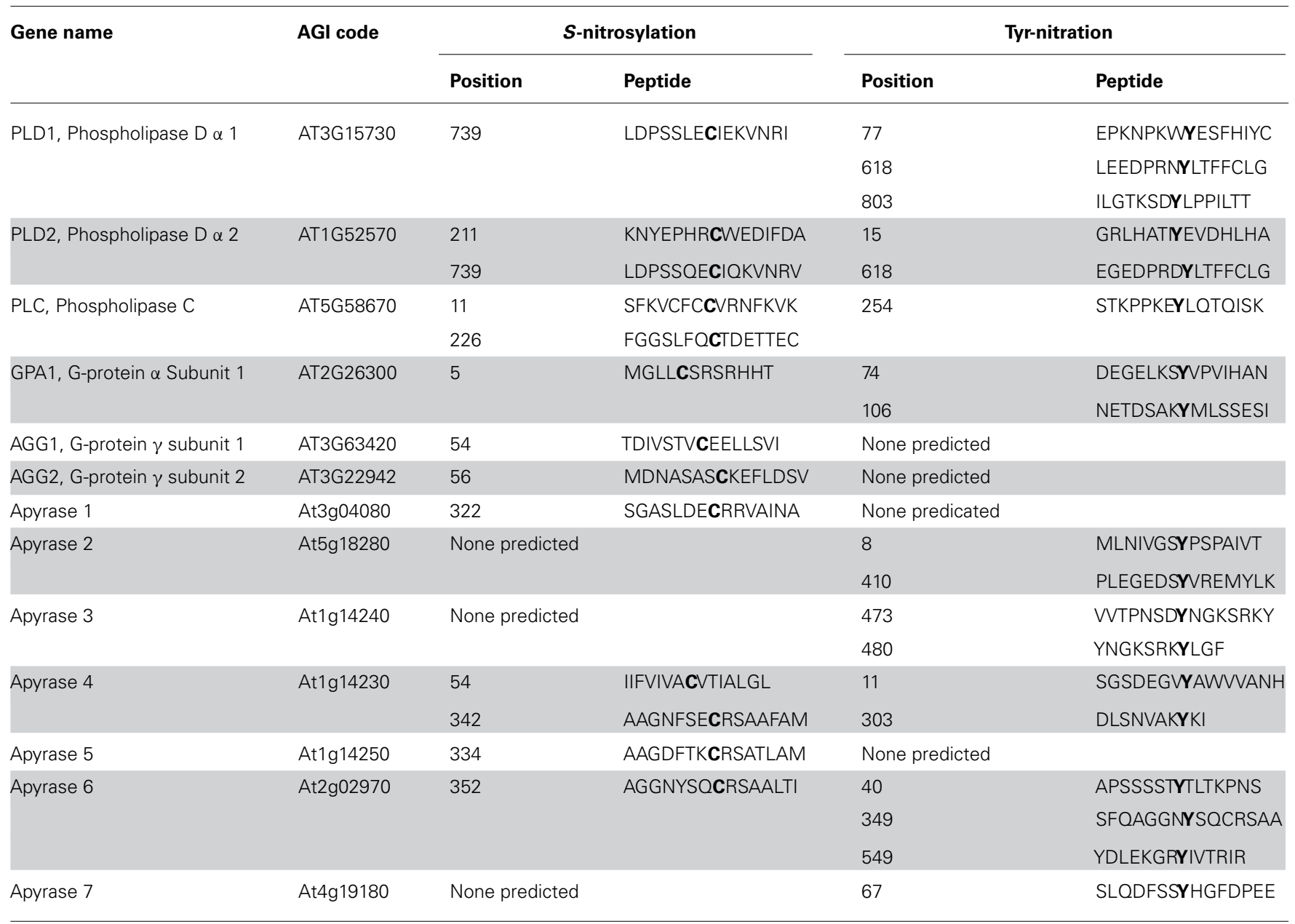

Proteins AGB1 and GCR1 are not included because neither modification was predicated at any residue.

The position of the cysteine or tyrosine amino acid predicted to be modified is given and indicated in bold. 
and Rampitsch (2013) suggest that these NO-dependent changes might serve to reversibly occupy an amino acid that could be otherwise modified in a way that would lead to a different activity change (i.e., phosphorylation, carbonylation, or disulfide bond formation).

Predictive, sequence-specific models have been developed to identify sites of cysteine-nitrosylation (Xue et al., 2010) and tyrosine nitration (Liu et al., 2011). These algorithms were developed from K-means clustering methods, and can be used with different thresholds of reliability, high/medium/low, based on the false discovery rate (FDR; 10\%/15\%/20\%). Like efforts to experimentally identify the protein modifications directly, these predictive models are new and still being optimized, but they can be used to identify potential targets of study for these regulatory modifications.

Apyrase proteins serve to regulate extracellular ATP concentration in animal cells (Plesner, 1995; Gaddie and Kirley, 2010), and a similar role may exist for these proteins in plant cells. The Arabidopsis nucleoside triphosphate-diphosphohydrolases termed apyrase 1 and 2 have been implicated in e-ATP signaling (Clark et al., 2011; Liu et al., 2012), although they may do so from a Golgi locale (Chiu et al., 2012; Schiller et al., 2012) rather than from a plasma membrane site. When ecto-apyrase activity is inhibited by antibodies raised to APY1 and APY2, the [eATP] of media in which pollen tubes are growing rises several fold and pollen tube growth is inhibited (Wu et al., 2007). Similarly, when APY1/APY2 expression is suppressed by RNAi in R2-4A mutants, this raises the [eATP] of the media and inhibits seedling growth (Salmi, Kim and Roux, unpublished). Although the expression/and or activity of APY1 and 2 appear to influence [eATP], and sites of [eATP] release in roots coincide with sites of increased expression of APY1 and APY2 (Roux et al., 2008), it is of course possible that the Golgi function of APY1 and APY2 could regulate growth independent of their influence on [eATP]. Theoretically, other members of the apyrase family could also help regulate [eATP]. At least one Tyr-nitration or $S$-nitrosylation site is predicted in the proteins encoded by each of the seven members of the Arabidopsis thaliana family of apyrase genes (Table 1; Yang et al., 2013), and these predictions should be experimentally evaluated.

Nitric oxide production is necessary for the cellular response to extracellular nucleotides (Clark et al., 2010, 2011). Similarly, respiratory burst oxidases also play a critical role in mediating plant responses to eATP (Suzuki et al., 2011). Moreover, the timing of

Table 2 | Computationally predicted NO mediated modifications of known auxin transport proteins (Xue et al., 2010; Liu et al., 2011). Only predictions included in the "high" threshold category are included here (10\% FDR).

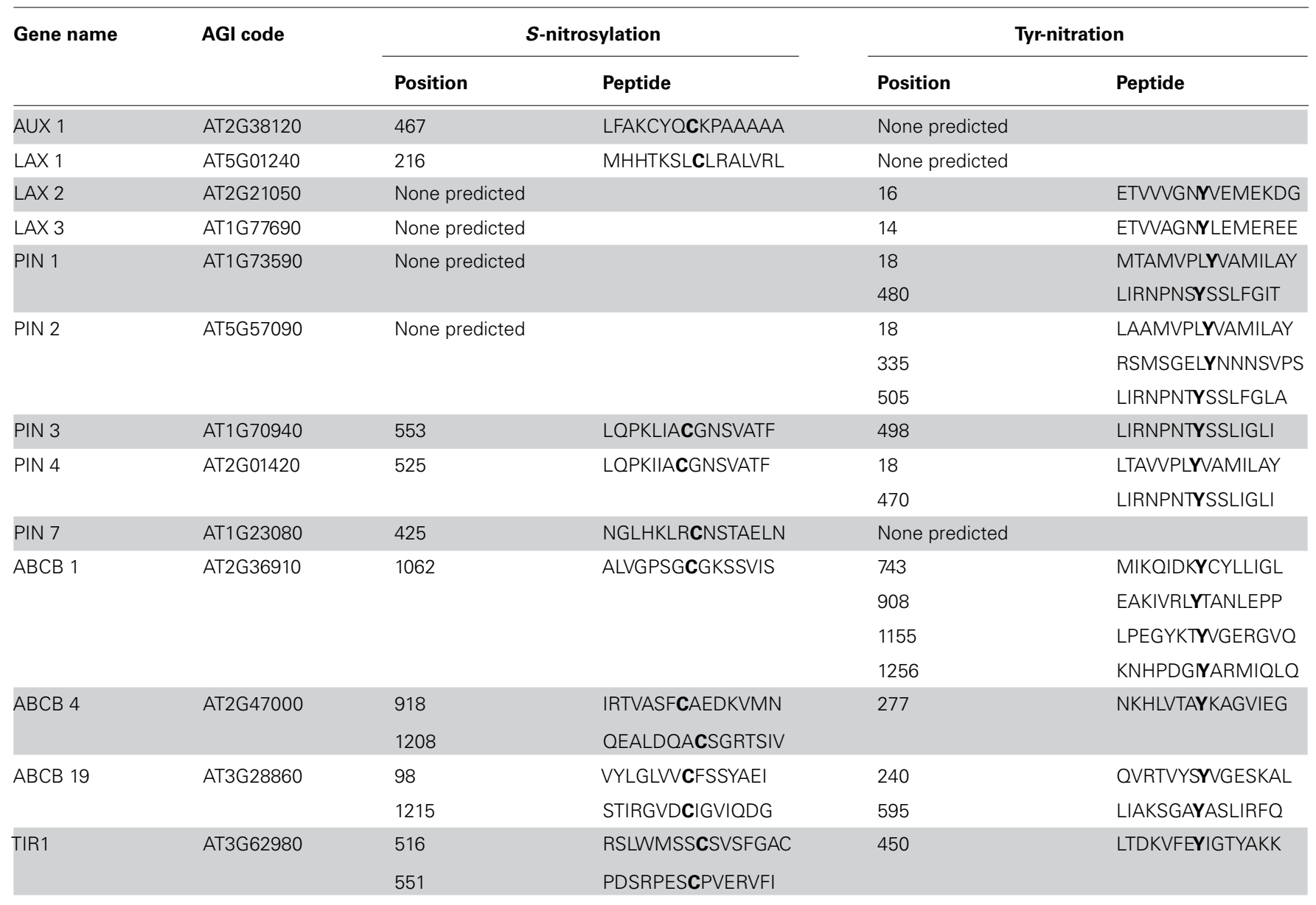

The position of the cysteine or tyrosine amino acid predicted to be modified is given and indicated in bold. 
e-ATP-induced production of NO and ROS is similar (both within 30-45 min; Clark et al., 2010, 2011). To the extent that NO and ROS are induced at about the same time and are both needed for plants to respond to eATP, these results suggest the possibility that peroxynitirite could serve to induce Tyr-nitration and thus serve as an important regulator of its own production in a feedback mechanism.

Peroxynitrite $\left(\mathrm{ONOO}^{-}\right)$is a potent oxidant and nitrating species that can be formed by the reaction of $\mathrm{NO}$ and $\mathrm{O}_{2}^{-}$when both of these signaling molecules are present at the same time in the same cell. Plant biologists are just beginning to assay the role of peroxynitrite in plant growth and development (Leitner et al., 2009; Arasimowicz-Jelonek and Floryszak-Wieczorek, 2011; Vandelle and Delledonne, 2011), and thus far it is implicated in hypersensitive defense responses (Saito et al., 2006; Leitner et al., 2009; Gaupels et al., 2011; Bellin et al., 2013) and root development and senescence (Gaupels et al., 2011; Begara-Morales et al., 2013). Peroxynitrite can oxidize proteins and membrane lipids causing cellular damage, and its formation is most likely controlled by the local production of superoxide (Vandelle and Delledonne, 2011). Through this peroxynitrite mechanism, oxidation of lipids may play a role in plant lipid signaling pathways (Sanchez-Calvo et al., 2013).

Could peroxynitrite help mediate some eATP effects? Chivasa et al. (2005) have proposed a role for eATP in programmed cell death, and there is evidence that peroxynitrite may help mediate programmed cell death in plant cells (Serrano et al., 2012a,b). eATP has also been implicated in plant pathogen responses (Chivasa et al., 2009), and a signaling role for $\mathrm{ONOO}^{-}$in these responses has been documented (Saito et al., 2006; Gaupels et al., 2011). More interesting is the potential role for $\mathrm{ONOO}^{-}$in plant growth responses to applied eATP. A growth-inhibiting concentration of eATP produces high levels of NO and ROS, while lower, growth-promoting concentrations of eATP induce low levels of NO and ROS (Clark et al., 2010). Both situations may lead to the production of peroxynitrite, which could mediate the growth regulatory effects of eATP.

Another important target for peroxynitrite-mediated nitration in animal cells is the second messenger cyclic guanosine monophosphate (cGMP; Akaike et al., 2010; Sawa et al., 2013).

\section{REFERENCES}

Akaike, T., Fujii, S., Sawa, T., and Ihara, H. (2010). Cell signaling mediated by nitrated cyclic guanine nucleotide. Nitric Oxide: Biol. Chem. 23, 166174. doi: 10.1016/j.niox.2010.06.006 Arasimowicz-Jelonek, M., and Floryszak-Wieczorek, J. (2011). Understanding the fate of peroxynitrite in plant cells: from physiology to pathophysiology. Phytochemistry 72, 681-688. doi: 10.1016/j. phytochem.2011.02.025

Astier, J., and Lindermayr, C. (2012). Nitric oxide-dependent posttranslational modification in plants: an update. Int. J. Mol. Sci. 13, 15193 15208. doi: 10.3390/ijms131115193
Begara-Morales, J. C., Chaki, M., Sanchez-Calvo, B., Mata-Perez, C. Leterrier, M., Palma, J. M., et al. (2013). Protein tyrosine nitration in pea roots during development and senescence. J. Exp. Bot. 64, 11211134. doi: 10.1093/jxb/ert006

Bellin, D., Asai, S., Delledonne, M., and Yoshioka, H. (2013). Nitric oxide as a mediator for defense responses. $\mathrm{Mol}$. Plant Microbe Interact. 26, 271-277. doi: 10.1094/MPMI-09-12-0214-CR

Besson-Bard, A., Pugin, A., and Wendehenne, D. (2008). New insights into nitric oxide signaling in plants. Annu. Rev. Plant Biol. 59, 21-39. doi: 10.1146/annurev.arplant.59.032607. 092830

Recently, a role of nitrated cGMP (8-nitro-cGMP) in Abscisic acid (ABA)-induced stomatal closing was discovered (Joudoi et al., 2013). Because ABA-induced stomatal closure can be partially blocked by mammalian purinoceptor antagonists (Clark et al., 2011), and treating Arabidopsis leaves with high levels of eATP induces both NO and ROS, it will be important to determine if eATP treatment causes nitration of cGMP in guard cells. Recently, cGMP was shown to promote lateral root formation in Arabidopsis by regulating polar auxin transport (Li and Jia, 2013). Thus, a plausible speculation is that nitration of cGMP might also play an important role in regulating auxin transport.

The effects of exogenously applied ATP and ATP analogs are pronounced in root development (Lew and Dearnaley, 2000; Tang et al., 2003; Wu and Wu, 2008; Wu et al., 2008). Proper localization of auxin is necessary for normal root development. In the apyrase mutants described by Liu et al. (2012) localization of several auxin transporters and the abundance of transcripts encoding these transporters were not altered in plants with inhibited auxin transport and stunted and altered root anatomy. One mechanism for this could be regulation of the transporter activity, and NOmediated PTMs are likely candidates for this regulation. Several proteins known to be involved in polar auxin transport have predicted $S$-nitrosylation and Tyr-nitration sites (Table 2), and these predictions should be experimentally evaluated.

\section{CONCLUSION AND FUTURE DIRECTIONS}

There is increasing evidence to support a role for NO-mediated PTMs of proteins in the regulation of plant cellular processes by eATP. Although numerous plant proteins have been predicted to undergo these changes and experimentally shown to have them in various conditions, the regulatory role of these PTMs remains to be demonstrated in all but a few cases. Given the central role of auxin in plant growth control, it is likely that the dramatic effects of extracellular nucleotides on auxin transport account for many of their effects on plant growth. Thus, a more complete understanding of how NO regulates auxin transport, whether by PTM of auxin transporters or by other mechanisms, will be key to clarifying why eATP-induced NO production is a necessary step in transducing extracellular nucleotide effects on plant growth and development.

Burnstock, G., Fredholm, B. B., North, R. A., and Verkhratsky, A. (2010). The birth and postnatal development of purinergic signalling. Acta Physiol. 199, 93-147. doi: 10.1111/j.17481716.2010.02114.x

Bykova, N. V., and Rampitsch, C. (2013). Modulating protein function through reversible oxidation: redox-mediated processes in plants revealed through proteomics. Proteomics 13, 579-596. doi: 10.1002/pmic.201200270

Chaki, M., Valderrama, R., FernandezOcana, A. M., Carreras, A., LopezJaramillo, J., Luque, F., et al. (2009). Protein targets of tyrosine nitration in sunflower (Helianthus annuus L.) hypocotyls. J. Exp. Bot. 60, 42214234. doi: 10.1093/jxb/erp263

Chiu, T. Y., Christiansen, K., Moreno, I., Lao, J. M., Loque, D., Orellana, A., et al. (2012). AtAPY1 and AtAPY2 function as golgi-localized nucleoside diphosphatases in Arabidopsis thaliana. Plant Cell Physiol. 53, 19131925. doi: $10.1093 / \mathrm{pcp} / \mathrm{pcs} 131$

Chivasa, S., Ndimba, B. K., Simon, W. J., Lindsey, K., and Slabas, A. R. (2005). Extracellular ATP functions as an endogenous external metabolite regulating plant cell viability. Plant Cell 17, 3019-3034. doi: 10.1105/tpc.105.036806

Chivasa, S., Murphy, A. M., Hamilton, J. M., Lindsey, K., Carr, J. P., 
and Slabas, A. R. (2009). Extracellular ATP is a regulator of pathogen defence in plants. Plant J. 60, 436448. doi: 10.1111/j.1365-313X.2009. 03968.x

Clark, G., Fraley, D., Steinebrunner, I., Cervantes, A., Onyirimba, J., Liu, A., etal. (2011). Extracellular nucleotides and apyrases regulate stomatal aperture in Arabidopsis. Plant Physiol. 156, 1740-1753. doi: 10.1104/pp.111.174466

Clark, G., and Roux, S. J. (2009). Extracellular nucleotides: ancient signaling molecules. Plant Sci. 177, 239-244. doi: 10.1016/j.plantsci.2009.05.004

Clark, G., and Roux, S. J. (2011). Apyrases, extracellular ATP and the regulation of growth. Curr. Opin. Plant Biol. 14, 700-706. doi: 10.1016/ j.pbi.2011.07.013

Clark, G., Wu, M., Wat, N., Onyirimba, J., Pham, T., Herz, N., et al. (2010) Both the stimulation and inhibition of root hair growth induced by extracellular nucleotides in Arabidopsis are mediated by nitric oxide and reactive oxygen species. Plant Mol. Biol. 74, 423-435. doi: 10.1007/s11103-010 9683-7

Corpas, F. J., Palma, J. M., Del Rio, L. A., and Barroso, J. B. (2013). Protein tyrosine nitration in higher plants grown under natural and stress conditions. Front. Plant Sci. 4:29. doi: 10.3389/fpls.2013.00029

Correa-Aragunde, N., Foresi, N., Delledonne, M., and Lamattina, L. (2013). Auxin induces redox regulation of ascorbate peroxidase 1 activity by $S$ nitrosylation/denitrosylation balance resulting in changes of root growth pattern in Arabidopsis. J. Exp. Bot. 64, 3339-3349. doi: 10.1093/jxb/ert172

Correa-Aragunde, N., Graziano, M., and Lamattina, L. (2004). Nitric oxide plays a central role in determining lateral root development in tomato. Planta 218, 900-905. doi: 10.1007/s00425-003-1172-7

D’Andrea, P., Romanello, M., Bicego, M., Steinberg, T. H., and Tell, G. (2008). $\mathrm{H}(2) \mathrm{O}(2)$ modulates purinergic-dependent calcium signalling in osteoblast-like cells. Cell Calcium 43, 457-468. doi: 10.1016/j.ceca.2007.07.007

Demidchik, V., Nichols, C., Oliynyk, M., Dark, A., Glover, B. J., and Davies, J. M. (2003). Is ATP a signaling agent in plants? Plant Physiol. 133, 456-461. doi: 10.1104/pp.103.024091

Demidchik, V., Shang, Z., Shin, R., Colaco, R., Laohavisit, A., Shabala, S., et al. (2011). Receptor-like activity evoked by extracellular ADP in Arabidopsis root epidermal plasma membrane. Plant Physiol. 156,
1375-1385. doi: 10.1104/pp.111.17 4722

Demidchik, V., Shang, Z., Shin, R. Thompson, E., Rubio, L., Laohavisit, A., etal. (2009). Plant extracellular ATP signalling by plasma membrane NADPH oxidase and $\mathrm{Ca}^{2+}$ channels. Plant J. 58, 903-913. doi: 10.1111/j.1365-313X.2009.03830.x

Fares, A., Rossignol, M., and Peltier, J. B. (2011). Proteomics investigation of endogenous $S$-nitrosylation in Arabidopsis. Biochem. Biophys. Res. Commun. 416, 331-336. doi: 10.1016/j.bbrc.2011.11.036

Feng, J., Wang, C., Chen, Q. G., Chen, H., Ren, B., Li, X. M., et al. (2013). S-nitrosylation of phosphotransfer proteins represses cytokinin signaling. Nat. Commun. 4, 1529. doi: 10.1038/ncomms2541

Fernandez-Marcos, M., Sanz, L., Lewis, D. R., Muday, G. K., and Lorenzo, O. (2011). Nitric oxide causes root apical meristem defects and growth inhibition while reducing PIN-FORMED 1 (PIN1)-dependent acropetal auxin transport. Proc. Natl. Acad. Sci. U.S.A. 108, 18506-18511. doi: 10.1073/pnas.1108644108

Foresi, N. P., Laxalt, A. M., Tonon, C. V., Casalongue, C. A., and Lamattina, L. (2007). Extracellular ATP induces nitric oxide production in tomato cell suspensions. Plant Physiol. 145, 589592. doi: 10.1104/pp.107.106518

Gaddie, K. J., and Kirley, T. L. (2010). Proline residues link the active site to transmembrane domain movements in human nucleoside triphosphate diphosphohydrolase 3 (NTPDase3). Purinergic Signal. 6, 327-337. doi: 10.1007/s11302-010-9180-0

Gaupels, F., Spiazzi-Vandelle, E., Yang, D., and Delledonne, M. (2011). Detection of peroxynitrite accumulation in Arabidopsis thaliana during the hypersensitive defense response. Nitric Oxide: Biol. Chem. 25, 222-228. doi: 10.1016/j.niox.2011. 01.009

Gout, E., Bligny, R., and Douce R. (1992). Regulation of intracellular ph values in higher-plant cells: c-13 and p-31 nuclear-magneticresonance studies. J. Biol. Chem. 267, 13903-13909.

Guo, K., Xia, K., and Yang, Z. M. (2008). Regulation of tomato lateral root development by carbon monoxide and involvement in auxin and nitric oxide. J. Exp. Bot. 59, 34433452. doi: 10.1093/jxb/ern194

Hao, L. H., Wang, W. X., Chen, C., Wang, Y. F., Liu, T., Li, X., et al. (2012). Extracellular ATP promotes stomatal opening of Arabidopsis thaliana through heterotrimeric
$G$ protein a subunit and reactive oxygen species. Mol. Plant 5, 852-864. doi: $10.1093 / \mathrm{mp} /$ ssr095

Harada, N. (2010). Role of nitric oxide on purinergic signalling in the cochlea. Purinergic Signal. 6, 211220. doi: 10.1007/s11302-010-9186-7

Jacques, S., Ghesquiere, B., Van Breusegem, F., and Gevaert, K (2013). Plant proteins under oxidative attack. Proteomics 13, 932-940. doi: 10.1002/pmic.201200237

Jaffrey, S. R., Erdjument-Bromage, $\mathrm{H}$. Ferris, C. D., Tempst, P., and Snyder S. H. (2001). Protein S-nitrosylation: a physiological signal for neuronal nitric oxide. Nat. Cell Biol. 3, 193 197. doi: 10.1038/35055104

Jeter, C. R., Tang, W. Q., Henaff, E., Butterfield, T., and Roux, S. J. (2004). Evidence of a novel cell signaling role for extracellular adenosine triphosphates and diphosphates in Arabidopsis. Plant Cell 16, 2652-2664. doi: 10.1105/tpc.104.023945

Joudoi, T., Shichiri, Y., Kamizono, N., Akaike, T., Sawa, T., Yoshitake, J., et al. (2013). Nitrated cyclic GMP modulates guard cell signaling in Arabidopsis. Plant Cell 25, 558-571. doi: 10.1105/tpc.112.105049

Khakh, B. S., and Burnstock, G. (2009). The double life of ATP. Sci. Am. 301, 84. doi: 10.1038/ scientificamerican1209-84

Kim, S.-Y., Sivaguru, M., and Stacey, G. (2006). Extracellular ATP in plants. Visualization, localization, and analysis of physiological significance in growth and signaling. Plant Physiol. 142, 984-992. doi: 10.1104/pp.106.085670

Knowles, A. F. (2011). The GDA1_CD39 superfamily: NTPDases with diverse functions. Purinergic Signal. 7, 21-45. doi: 10.1007/s11302-010-9214-7

Kolbert, Z., Peto, A., Lehotai, N., Feigl, G., and Erdei, L. (2012). Long-term copper $\left(\mathrm{Cu}^{2+}\right)$ exposure impacts on auxin, nitric oxide (NO) metabolism and morphology of Arabidopsis thaliana L. Plant Growth Regul. 68, 151-159. doi: 10.1007/s10725-012-9701-7

Kovacs, I., and Lindermayr, C. (2013). Nitric oxide-based protein modification: formation and sitespecificity of protein $S$-nitrosylation. Front. Plant Sci. 4:137. doi: 10.3389/fpls.2013.00137

Lanteri, M. L., Laxalt, A. M., and Lamattina, L. (2008). Nitric oxide triggers phosphatidic acid accumulation via phospholipase D during auxininduced adventitious root formation in cucumber. Plant Physiol. 147, 188198. doi: $10.1104 /$ pp.107.111815
Lanteri, M. L., Pagnussat, G. C., and Lamattina, L. (2006). Calcium and calcium-dependent protein kinases are involved in nitric oxide- and auxin-induced adventitious root formation in cucumber. J. Exp. Bot. 57, 1341-1351. doi: 10.1093/jxb/ erj109

Lazarowski, E. R., Boucher, R. C., and Harden, T. K. (2003). Mechanisms of release of nucleotides and integration of their action as $\mathrm{P} 2 \mathrm{X}-$ and P2Y-receptor activating molecules. Mol. Pharmacol. 64, 785-795. doi: 10.1124/mol.64.4.785

Lea, U. S., Ten Hoopen, F., Provan, F., Kaiser, W. M., Meyer, C., and Lillo, C. (2004). Mutation of the regulatory phosphorylation site of tobacco nitrate reductase results in high nitrite excretion and NO emission from leaf and root tissue. Planta 219, 59-65. doi: 10.1007/s00425-0041209-6

Leitner, M., Vandelle, E., Gaupels, F., Bellin, D., and Delledonne, M. (2009). NO signals in the haze nitric oxide signalling in plant defence. Curr. Opin. Plant Biol. 12, 451-458. doi: 10.1016/j.pbi.2009.05.012

Lew, R. R., and Dearnaley, J. D. W. (2000). Extracellular nucleotide effects on the electrical properties of growing Arabidopsis thaliana root hairs. Plant Sci. 153, 1-6. doi: 10.1016/S0168-9452(99)00242-3

Li, J. S., and Jia, H. L. (2013). cGMP modulates Arabidopsis lateral root formation through regulation of polar auxin transport. Plant Physiol. Biochem. 66, 105117. doi: 10.1016/j.plaphy.2013. 02.014

Liao, W. B., Huang, G. B., Yu, J. H., Zhang, M. L., and Shi, X. L. (2011). Nitric oxide and hydrogen peroxide are involved in indole-3-butyric acid-induced adventitious root development in marigold. J. Hortic. Sci. Biotechnol. 86, 159-165.

Lindermayr, C., Saalbach, G., and Durner, J. (2005). Proteomic identification of $S$-nitrosylated proteins in Arabidopsis. Plant Physiol. 137, 921-930. doi: 10.1104/pp.104.05 8719

Liu, X., Wu, J., Clark, G., Lundy, S., Lim, M., Arnold, D., et al. (2012). Role for Apyrases in polar auxin transport in Arabidopsis. Plant Physiol. 160, 1985-1995. doi: 10.1104/pp.112. 202887

Liu, Z., Cao, J., Ma, Q., Gao, X., Ren, J., and Xue, Y. (2011). GPSYNO2: computational prediction of tyrosine nitration sites in proteins. Mol. Biosyst. 7, 1197-1204. doi: 10.1039/c0mb00279h 
Lozano-Juste, J., Colom-Moreno, R., and Leon, J. (2011). In vivo protein tyrosine nitration in Arabidopsis thaliana. J. Exp. Bot. 62, 3501-3517. doi: 10.1093/jxb/err042

Pagnussat, G. C., Lanteri, M. L., and Lamattina, L. (2003). Nitric oxide and cyclic GMP are messengers in the indole acetic acidinduced adventitious rooting process. Plant Physiol. 132, 1241-1248. doi: 10.1104/pp.103.022228

Pagnussat, G. C., Lanteri, M. L., Lombardo, M. C., and Lamattina, L. (2004). Nitric oxide mediates the indole acetic acid induction activation of a mitogen-activated protein kinase cascade involved in adventitious root development. Plant Physiol. 135, 279-286. doi: 10.1104/pp.103.038554

Peto, A., Lehotai, N., Lozano-Juste, J., Leon, J., Tari, I., Erdei, L., et al. (2011). Involvement of nitric oxide and auxin in signal transduction of copper-induced morphological responses in Arabidopsis seedlings. Ann. Bot. 108, 449-457. doi: 10.1093/aob/mcr176

Plesner, L. (1995). Ecto-atpases: identities and functions. Int. Rev. Cytol. Surv. Cell Biol. 158, 141-214. doi: 10.1016/S0074-7696(08)62487-0

Reichler, S. A., Torres, J., Rivera, A. L., Cintolesi, V. A., Clark, G., and Roux, S. J. (2009). Intersection of two signalling pathways: extracellular nucleotides regulate pollen germination and pollen tube growth via nitric oxide. J. Exp. Bot. 60, 2129-2138. doi: 10.1093/jxb/ erp091

Roux, S., Wu, J., Henaff, E., Torres, J., and Clark, G. (2008). Regions of growth are regions of highest release of ATP and highest expression of ectonucleotidases AtAPY1 and AtAPY2 in Arabidopsis. Purinergic Signal. 4, S112.

Roux, S. J., and Steinebrunner, I. (2007). Extracellular ATP: an unexpected role as a signaler in plants. Trends Plant Sci. 12, 522-527. doi: 10.1016/j.tplants.2007.09.003

Saito, S., Yamamoto-Katou, A., Yoshioka, H., Doke, N., and Kawakita, K. (2006). Peroxynitrite generation and tyrosine nitration in defense responses in tobacco BY-2 cells. Plant Cell Physiol. 47, 689-697. doi: 10.1093/pcp/pcj038

Sanchez-Calvo, B., Barroso, J. B., and Corpas, F. J. (2013). Hypothesis: nitro-fatty acids play a role in plant metabolism. Plant Sci. 199, 1-6. doi: 10.1016/j.plantsci.2012.10.006

Sawa, T., Ihara, H., Ida, T., Fujii, S., Nishida, M., and Akaike, T.
(2013). Formation, signaling functions, and metabolisms of nitrated cyclic nucleotide. Nitric Oxide 34, 10-18. doi: 10.1016/j.niox.2013.04. 004

Schiller, M., Massalski, C., Kurth, T., and Steinebrunner, I. (2012). The Ara bidopsis apyrase AtAPY1 is localized in the Golgi instead of the extracellular space. BMC Plant Biol. 12:123. doi: 10.1186/1471-2229-12-123

Serrano, I., Romero-Puertas, M. C. Rodriguez Serrano, M., Sandalio, L. M., and Olmedilla, A. (2012a). Role of peroxynitrite in programmed cell death induced in self-incompatible pollen. Plant Signal. Behav. 7, 779781. doi: 10.4161/psb.20570

Serrano, I., Romero-Puertas, M. C., Rodriguez-Serrano, M., Sandalio, L. M., and Olmedilla, A. (2012b). Peroxynitrite mediates programmed cell death both in papillar cells and in self-incompatible pollen in the olive (Olea europaea L.). J. Exp. Bot. 63, 1479-1493. doi: 10.1093/jxb/err392

She, X. P., and Song, X. G. (2006). Cytokinin- and auxin-induced stomatal opening is related to the change of nitric oxide levels in guard cells in broad bean. Physiol. Plant. 128, 569-579. doi: 10.1111/j.13993054.2006.00782.x

Simontacchi, M., Garcia-Mata, C., Bartoli, C. G., Santa-Maria, G. E., and Lamattina, L. (2013). Nitric oxide as a key component in hormoneregulated processes. Plant Cell Rep. 32, 853-866. doi: 10.1007/s00299013-1434-1

Song, C. J., Steinebrunner, I., Wang, X. Z., Stout, S. C., and Roux, S. J. (2006). Extracellular ATP induces the accumulation of superoxide via NADPH oxidases in Arabidopsis. Plant Physiol. 140, 1222-1232. doi: 10.1104/pp.105.073072

Sueldo, D. J., Foresi, N. P., Casalongue, C. A., Lamattina, L., and Laxalt, A. M. (2010). Phosphatidic acid formation is required for extracellular ATP-mediated nitric oxide production in suspension-cultured tomato cells. New Phytol. 185, 909-916. doi: 10.1111/j.1469-8137.2009.03165.x

Sun, J., Zhang, X., Deng, S. R., Zhang, C. L., Wang, M. J., Ding, M. Q., et al. (2012). Extracellular ATP signaling is mediated by $\mathrm{H}_{2} \mathrm{O}_{2}$ and cytosolic $\mathrm{Ca}^{2+}$ in the salt response of populus euphratica cells. PLoS ONE 7:e53136. doi: 10.1371/journal.pone.0053136

Suzuki, N., Miller, G., Morales, J., Shulaev, V., Torres, M. A., and Mittler, R. (2011). Respiratory burst oxidases: the engines of ROS signaling. Curr. Opin. Plant Biol. 14, 691-699. doi: 10.1016/j.pbi.2011.07.014
Tanaka, K., Gilroy, S., Jones, A. M., and Stacey, G. (2010). Extracellular ATP signaling in plants. Trends Cell Biol. 20, 601-608. doi: 10.1016/j.tcb.2010.07.005

Tang, W. Q., Brady, S. R., Sun, Y., Muday, G. K., and Roux, S. J. (2003). Extracellular ATP inhibits root gravitropism at concentrations that inhibit polar auxin transport. Plant Physiol. 131, 147-154. doi: 10.1104/pp.013672

Terrile, M. C., Paris, R., CalderonVillalobos, L. I. A., Iglesias, M. J., Lamattina, L., Estelle, M., et al. (2012). Nitric oxide influences auxin signaling through $S$-nitrosylation of the Arabidopsis TRANSPORT INHIBITOR RESPONSE 1 auxin receptor. Plant J. 70, 492-500. doi: 10.1111/j.1365-313X.2011.04885.x

Tonon, C., Cecilia Terrile, M., Jose Iglesias, M., Lamattina, L., and Casalongue, C. (2010). Extracellular ATP, nitric oxide and superoxide act coordinately to regulate hypocotyl growth in etiolated Arabidopsis seedlings. J. Plant Physiol. 167, 540-546. doi: 10.1016/j.jplph.2009.11.002

Vandelle, E., and Delledonne, M. (2011). Peroxynitrite formation and function in plants. Plant Sci. 181, 534-539. doi: 10.1016/j.plantsci.2011.05.002

Weerasinghe, R. R., Swanson, S. J., Okada, S. F., Garrett, M. B., Kim, S.-Y., Stacey, G., et al. (2009). Touch induces ATP release in Arabidopsis roots that is modulated by the heterotrimeric G-protein complex. FEBS Lett. 583, 2521-2526. doi: 10.1016/j.febslet.2009.07.007

Wu, J., Steinebrunner, I., Sun, Y., Butterfield, T., Torres, J., Arnold, D. et al. (2007). Apyrases (nucleoside triphosphate-diphosphohydrolases) play a key role in growth control in Arabidopsis. Plant Physiol. 144, 961-975. doi: 10.1104/pp.107.097568

Wu, S.-J., and Wu, J.-Y. (2008). Extracellular ATP-induced NO production and its dependence on membrane $\mathrm{Ca}^{(2+)}$ flux in Salvia miltiorrhiza hairy roots. J. Exp. Bot. 59, $4007-$ 4016. doi: 10.1093/jxb/ern242

Wu, S. J., Liu, Y. S., and Wu, J. Y. (2008). The signaling role of extracellular ATP and its dependence on $\mathrm{Ca}^{(2+)}$ flux in elicitation of Salvia miltiorrhiza hairy root cultures. Plant Cell Physiol. 49, 617-624. doi: 10.1093/pcp/pcn033

Xu, J., Wang, W. Y., Sun, J. H., Zhang, Y., Ge, Q., Du, L. G. et al. (2011). Involvement of auxin and nitric oxide in plant Cd-stress responses. Plant Soil 346, 107-119. doi: 10.1007/s11104-011-0800-4
Xue, Y., Liu, Z., Gao, X., Jin, C., Wen, L., Yao, X., et al. (2010). GPSSNO: computational prediction of protein $S$-nitrosylation sites with a modified GPS algorithm. PLoS ONE 5:e11290. doi: 10.1371/journal.pone. 0011290

Yadav, S., David, A., and Bhatla, S. C. (2011). Nitric oxide accumulation and actin distribution during auxin-induced adventitious root development in sunflower. Sci. Hortic. 129, 159-166. doi: 10.1016/j.scienta.2011.03.030

Yang, J., Wu, J., Romanovicz, D., Clark, G., and Roux, S. J. (2013). Coregulation of exine wall patterning, pollen fertility and anther dehiscence by Arabidopsis apyrases 6 and 7. Plant Physiol. Biochem. 69, 62-73. doi: 10.1016/j.plaphy.2013.04.022

Yoshioka, H., Mase, K., Yoshioka, M., Kobayashi, M., and Asai, S. (2011). Regulatory mechanisms of nitric oxide and reactive oxygen species generation and their role in plant immunity. Nitric Oxide: Biol. Chem. 25, 216-221. doi: 10.1016/j.niox.2010.12.008

Yun, B. W., Feechan, A., Yin, M. H., Saidi, N. B. B., Le Bihan, T., Yu, M., et al. (2011). S-nitrosylation of NADPH oxidase regulates cell death in plant immunity. Nature 478, 264-268. doi: 10.1038/nature 10427

Conflict of Interest Statement: The authors declare that the research was conducted in the absence of any commercial or financial relationships that could be construed as a potential conflict of interest.

Received: 28 June 2013; accepted: 09 October 2013; published online: 25 October 2013.

Citation: Salmi ML, Clark G and Roux SJ (2013) Current status and proposed roles for nitric oxide as a key mediator of the effects of extracellular nucleotides on plant growth. Front. Plant Sci. 4:427. doi: 10.3389/fpls.2013.00427

This article was submitted to Plant Physiology, a section of the journal Frontiers in Plant Science.

Copyright (c) 2013 Salmi, Clark and Roux. This is an open-access article distributed under the terms of the Creative Commons Attribution License (CC BY). The use, distribution or reproduction in other forums is permitted, provided the original author(s) or licensor are credited and that the original publication in this journal is cited, in accordance with accepted academic practice. No use, distribution or reproduction is permitted which does not comply with these terms. 